\title{
The structure of g-digroup actions and representation theory*
}

\section{J. G. Rodríguez-Nieto, O. P. Salazar-Díaz, and R. Velásquez}

\author{
Communicated by A. V. Zhuchok
}

\begin{abstract}
The aim of this paper is to propose two possible ways of defining a g-digroup action and a first approximation to representation theory of g-digroups.
\end{abstract}

\section{Introduction}

Lie's third theorem asserts the existence of a bijection between local Lie groups and its finite dimensional Lie algebra in the following way: the tangent space at the identity of any Lie group is a Lie algebra, and also, that to any finite dimensional Lie algebra over the real or the complex numbers, corresponds the tangent space of a connected Lie group unique up to finite coverings. On the other hand, we have the so called Leibniz algebra introduced by A. M. Bloh in [1] and later rediscovered by Loday in 1993 in [9]. A Leibniz algebra is a non associative $\mathbb{K}$-algebra $M$ endowed with a bracket product $[\cdot, \cdot]$ that satisfies the Leibniz identity,

$$
[x,[y, z]]=[[x, y], z]+[y,[x, z]],
$$

${ }^{*}$ The first and the second author were supported by the Hermes project: Algunos aspectos algebraicos y geométricos de los digrupos generalizados, code 45519. The third author was supported by the CIEN research project: Sobre el segundo grupo de cohomología en superalgebras de Jordan, code 2019-26870.

2020 MSC: 20B10, 20B30, 20C99, 20K99, 20M10, 20M18, 20M30, 20 N99.

Key words and phrases: digroups, groups, semigroups, Abelian digroups, symmetric digroups, actions and representations. 
for every $x, y, z \in M$. When the bracket product is skew-symmetric the Leibniz identity becomes the Jacobi identity, therefore $M$ adopts a Lie algebra structure. Conversely any Lie algebra is obviously a Leibniz algebra.

Following the idea of a possible extension of Lie's third theorem for Leibniz algebras, J. L. Loday proposed the so called Coquecigrue problem, which consists in finding an appropriate structure that generalizes the concept of Lie group and whose algebra is the corresponding Leibniz algebra. A crucial aspect to consider for a possible solution to the previous problem is to determine the correct generalization of the concept of group. The hypothetical structure for the Leibniz case has been called Leibniz group, Loday group, or Coquecigrue.

Further results on the Coquecigrue problem and the third Lie Theorem are given by Monterde and Ongay [11]. The first approximation to the solution of the Coquecigrue problem was proposed independently by M. Kinyon [7], R. Felipe [4], and K. Liu [8], which is a generalization of the group structure with two products and it has been called a digroup. Digroups form an important variety of algebras arising from dimonoids introduced by Loday [9]. We refer the reader to the literature [18], [20], [19] and [21] for some results on dimonoids.

Besides, in [17] O. Salazar-Díaz, R. Velásquez and L. A. Wills-Toro studied a slightly different structure, allowing non-bilateral inverses, called generalized digroup, that from now on we call g-digroup.

Solutions to the Coquecigrue problem were given by S. Coves [3] (local), J. Mostovoy (categorical) [12], and M. Bordemann and F. Wagemann (Augmented Leibniz algebras) [2].

Due to the nature of the extension of groups to g-digroups, one might think that many definitions and results on group theory can be directly extended to g-digroups, see [14] and [15] in which we extend the concepts of free groups and order and we introduce the concept of tensor product, however, unexpected results could decline the balance, for example, in [15] we proved that Lagrange's theorem is not always true for g-digroups, so we have proposed some variants of Sylows's theorems. Following this line of extending results, in the current paper we introduce, the concept of classical action of g-digroups from two points of view, one is by considering a connection with the representation theory of $g$-digroups stated in [14], the other one is by extending the definition of action for digroups given in [5]. The latter way is not natural and the orbits are not well defined, therefore a theorem like Burnside's formula is not achieved yet. 
This paper is organized as follows. In section 2, we recall the concepts of g-digroups, g-subdigroups, free g-digroups and the symmetric g-digroup following papers [17], [14] and [15]. In section 3 we introduce the concept of classical action of g-digroups which is a natural extension of the one given in group theory, we prove Burnside's formula for g-digroup actions and a version of Cayley's theorem, weaker than the one given in [15], orbit spaces are also introduced. We end the section with an extension of the notion of digroup action, introduced by H. Guzman and F. Ongay in [5], to the generalized case, and we use this to get a first approach to representation theory for g-digroups. Such definition of action does not give a construction of orbits like in the group theory case.

\section{2. g-digroups}

In this section we give a short review of some definitions and results about g-digroups, for a deeper study see [14] and [17].

Definition 1. A set $D$ is called a g-digroup (generalized digroup) if it has two binary operations $\vdash$ and $\dashv$ over $D$, which are associative (each separately), and satisfy the conditions:

1) $x \vdash(y \dashv z)=(x \vdash y) \dashv z$

2) $x \dashv(y \dashv z)=x \dashv(y \vdash z)$, $(x \vdash y) \vdash z=(x \dashv y) \vdash z$

3) There exists (at least) an element $e$ in $D$, such that for all $x$ in $D$, $x \dashv e=x=e \vdash x$.

The elements that satisfy this condition are called bar-units and the set of bar-units in $D$, denoted by $E$ (or $E_{D}$ ), is called the halo of $D$.

4) For the fixed bar-unit $e$, we have that for each $x$ in $D$ there exist $x_{r_{e}}^{-1}$ and $x_{l_{e}}^{-1}$ in $D$ (the right-inverse of $x$ and the left-inverse of $x$, respectively) such that $x \vdash x_{r_{e}}^{-1}=e$ and $x_{l_{e}}^{-1} \dashv x=e$.

Let $\xi \in E$ be a bar-unit. We define the sets of left and right inverses, denoted by $G_{l}^{\xi}$ and $G_{r}^{\xi}$, respectively, as follows

$$
G_{l}^{\xi}=\left\{x_{l_{\xi}}^{-1} \mid x \in D\right\} \quad \text { and } \quad G_{r}^{\xi}=\left\{x_{r_{\xi}}^{-1} \mid x \in D\right\} .
$$

It is not hard to prove that $\left(G_{l}^{\xi}, \dashv\right)$ and $\left(G_{r}^{\xi}, \vdash\right)$ are isomorphic groups with identity $\xi$, see [17, Theorem 2]. A g-digroup is called trivial if it consists of only bar units.

The following proposition summarizes some basic and important properties of the binary operations $\dashv$ and $\vdash$. Its proof can be found in [17]. 
Proposition 1 ([17]). Let $D$ be a g-digroup and a fixed bar unit $\xi$. Then, for all $x, y$ in $D$,

(1) Given $x \in D$ and $\xi \in E$, we have that

$$
\begin{aligned}
& \left(x_{l_{\xi}}^{-1}\right)_{l_{\xi}}^{-1}=\left(x_{r_{\xi}}^{-1}\right)_{l_{\xi}}^{-1}=\xi \dashv x \\
& \left(x_{r_{\xi}}^{-1}\right)_{r_{\xi}}^{-1}=\left(x_{l_{\xi}}^{-1}\right)_{r_{\xi}}^{-1}=x \vdash \xi .
\end{aligned}
$$

(2) For all $x, y \in D$ and for every $\xi, \eta \in E$, we have that $y \dashv x_{r_{\xi}}^{-1}=y \dashv$ $x_{l_{\eta}}^{-1}$ and $x_{l_{\xi}}^{-1} \vdash y=x_{r_{\eta}}^{-1} \vdash y$.

(3) The inverse of the products are $(x \vdash y)_{l_{\xi}}^{-1}=(x \dashv y)_{l_{\xi}}^{-1}=y_{l_{\xi}}^{-1} \dashv x_{l_{e}}^{-1}$ and $(x \vdash y)_{r_{\xi}}^{-1}=(x \dashv y)_{r_{\xi}}^{-1}=y_{r_{\xi}}^{-1} \vdash x_{r_{e}}^{-1}$.

Since the proof of the following theorem comes from the results given in [17], we omit it.

Theorem 1. Let $(D, \vdash,-)$ be a g-digroup. For any $\xi, \zeta \in E$

a) $G_{l}^{\xi}=\xi \dashv G_{l}^{\zeta}$ and $G_{r}^{\xi}=G_{r}^{\zeta} \vdash \xi$,

b) $G_{l}^{\xi} \cong G_{l}^{\zeta} \cong G_{r}^{\xi} \cong G_{r}^{\zeta}$,

c) $\xi \dashv D=G_{l}^{\xi}$ and $D \vdash \xi=G_{r}^{\xi}$, which implies $G_{l}^{\xi} \dashv D=G_{l}^{\xi}$ and $D \vdash G_{r}^{\xi}=G_{r}^{\xi}$.

d) $E$ is a $G_{l}^{\xi}$-set respect to the action defined by $(a, \zeta) \mapsto a \bullet l \zeta:=a \vdash$ $\zeta \dashv a^{-1}$, for all $a \in G_{l}^{\xi}$ and $\zeta \in E$.

As it is shown in [17], $D$ can be characterized as

$$
D=\bigcup_{\xi \in E}^{\bullet} G_{l}^{\xi}=\bigcup_{\xi \in E}^{\bullet} G_{r}^{\xi}
$$

Let's recall that if $D$ and $D^{\prime}$ are g-digroups, a map $\phi: D \rightarrow D^{\prime}$ is a g-digroup homomorphism if for any $x, y \in D$

$$
\phi(x \dashv y)=\phi(x) \dashv \phi(y) \quad \text { and } \quad \phi(x \vdash y)=\phi(x) \vdash \phi(y) .
$$

In addition, if $\phi$ is a bijection, then $\phi$ is a g-digroup isomorphism.

The upcoming statement not only describes a way to construct gdigroups but also motivates a second characterization of g-digroups, that is an extension of the results of M. Kinyon (see [7]) and F. Ongay (see [13]). Its proof can be found in [17]. 
Theorem 2. Let $D$ be a g-digroup, let $E$ be the set of bar units and let $G_{l}^{\xi}$ be the set of left inverses respect to some $\xi \in E$. Then $G_{l}^{\xi} \times E$ is a g-digroup, with operations

$$
(a, \alpha) \vdash(b, \beta):=(a \dashv b, a \bullet \beta) \text { and }(a, \alpha) \dashv(b, \beta):=(a \dashv b, \alpha),
$$

isomorphic to $D$ respect to the isomorphism $\varphi_{l}: D \longrightarrow G_{l}^{\xi} \times E$, defined by $\varphi_{l}(x)=\left(\xi \dashv x, x \dashv x_{l_{\xi}}^{-1}\right)$, with inverse $\varphi_{l}^{-1}: G_{l}^{\xi} \times E \longrightarrow D$, given by $(a, \alpha) \mapsto \alpha \dashv a$.

Theorem 2 and formulas (2) establish a bijective correspondence between g-digroups and $G$-sets. This correspondence is easily understood because of the fact that if $E$ is a $G$-set, then $G \times E$ becomes the g-digroup $(G \times E, \vdash, \dashv)$, with a little modification of the binary operations given in (2) as follows. For any $(w, \xi)$ and $(u, \eta)$ in $G \times E$,

$$
(w, \xi) \vdash(u, \eta)=(w u, w \bullet \eta) \text { and }(w, \xi) \dashv(u, \eta)=(w u, \xi) .
$$

Conversely, any g-digroup $D$ can be uniquely splitted, up to isomorphisms, as a cartesian product $G_{l}^{\xi} \times E_{D}$ in such a way that its halo is the set $\{\xi\} \times E_{D}$. Respect to this decomposition of g-digroups, next theorem describes how the respective g-digroup homomorphisms are affected under such kind of factorization, its proof is done in [17].

Theorem 3. Let $\Psi: D \longrightarrow D^{\prime}$ be a g-digroup homomorphism. Then, there exists an unique homomorphism $\Psi^{\prime}: G_{l}^{\xi} \times E \longrightarrow G_{l}^{\xi^{\prime}} \times E^{\prime}$ such that the diagram

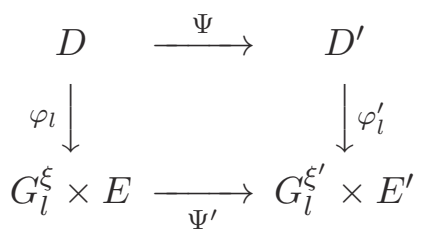

commutes, where $\Psi^{\prime} \equiv(\varphi, \mu)$, with

a) the $\operatorname{map} \varphi: G_{l}^{\xi} \longrightarrow G_{l}^{\xi^{\prime}}$, where $\varphi(a)=\xi^{\prime} \dashv \Psi(a)$, is a group homomorphism.

b) the map $\mu: E \longrightarrow E^{\prime}$, defined as $\mu(\alpha)=\Psi(\alpha)$ is an equivariant map, i.e.

$$
\mu(x \bullet \alpha)=\Psi(x) \bullet \mu(\alpha) \text { and } \mu(a \bullet \alpha)=\varphi(a) \bullet \mu(\alpha),
$$

for all $\alpha \in E$, all $x \in D$ and all $a \in G_{l}^{\xi}$. 
In this way, g-digroup homomorphisms $\Psi: G \times E \rightarrow G^{\prime} \times E^{\prime}$ between g-digroups can be described as $\Psi=(\varphi, \lambda)$, where $\varphi: G \rightarrow G^{\prime}$ is a group homomorphism and $\lambda: E \rightarrow E^{\prime}$ is an equivariant function.

Theorem 3 extends the correspondence between g-digroups and $G$-sets to a bijective correspondence between categories.

Definition 2. A subset $H$ of a g-digroup $(D ; \vdash, \dashv)$ is said to be a $g$ subdigroup of $D$, denoted by $H \leqslant D$, if $H$ with the restricted operations $\left.\vdash\right|_{H}$ and $\dashv \|_{H}$ to $H$, is itself a g-digroup.

It is not hard to prove that the set of bar units of a g-subdigroup $H$ of a g-digroup $D$ is $E_{H}=E \cap H$, where $E$ is the set of bar units of $D$, and the groups of left and right inverses for any $\xi \in E_{H}$, are $\Gamma_{l}^{\xi}=\left(G_{l}^{\xi} \cap H\right) \leqslant G_{l}^{\xi}$ and $\Gamma_{r}^{\xi}=\left(G_{r}^{\xi} \cap H\right) \leqslant G_{r}^{\xi}$, respectively. Moreover, $H \leqslant D$ iff there exist $\Gamma \leqslant G_{l}^{\xi}$ and an invariant $\Gamma$-set $\Delta \subset E(\Gamma \bullet \Delta=\Delta)$, such that $H \cong \Gamma \times \Delta$ (see [17, Lemma 6] for more details).

The following definition was given in [14].

Definition 3. Let $X$ be a subset of a g-digroup $(D, \vdash, \dashv)$. For $X^{-}$we mean the set of all inverses, right and left, with respect to all bar units in $D$ of all elements in $X$. In other words, if $E$ denotes the halo of $D$,

$$
X^{-}=X_{l}^{-} \cup X_{r}^{-},
$$

where $X_{l}^{-}:=\bigcup_{e \in E}\left\{x_{l_{e}}^{-1} \mid x \in X\right\}$ and $X_{r}^{-}:=\bigcup_{e \in E}\left\{x_{r_{e}}^{-1} \mid x \in X\right\}$.

The g-subdigroup of $D$ generated by $X$, denoted by $\langle X\rangle$, is the set of all elements of $D$ of the form

$$
\left(g_{1} \vdash \cdots \vdash g_{p}\right) \vdash y \dashv\left(h_{1} \dashv \cdots \dashv h_{k}\right),
$$

where $g_{t}, h_{n}$ and $y$ are in $X^{ \pm}=X \cup X^{-}$, for every $t=1,2, \ldots, p$ and $n=1,2, \ldots, k$.

As in [10], we denote the word (4) in $\langle X\rangle$ by $g_{1} \cdots g_{p} \breve{y} h_{1} \cdots h_{k}$. In this way

$$
g_{1} \vdash \cdots \vdash g_{k}=g_{1} \cdots g_{k-1} \breve{g}_{k} \text { and } g_{1} \dashv \cdots \dashv g_{k}=\breve{g}_{1} \cdots g_{k-1} g_{k} .
$$

It is not hard to prove that

$$
\begin{aligned}
& g_{1} \cdots g_{p} \breve{y} h_{1} \cdots h_{k} \vdash u_{1} \cdots g_{t} \breve{x} v_{1} \cdots v_{s} \\
& \quad=g_{1} \cdots g_{p} y h_{1} \cdots h_{k} u_{1} \cdots g_{t} \breve{x} v_{1} \cdots v_{s}
\end{aligned}
$$


and

$$
\begin{aligned}
& g_{1} \cdots g_{p} \breve{y} h_{1} \cdots h_{k} \dashv u_{1} \cdots g_{t} \breve{x} v_{1} \cdots v_{s} \\
& \quad=g_{1} \cdots g_{p} \breve{y} h_{1} \cdots h_{k} u_{1} \cdots g_{t} x v_{1} \cdots v_{s} .
\end{aligned}
$$

Let $X$ be a non empty set and $F(X)$ the free group generated by $X$, this is, the set of all words in $X^{ \pm}$. See [6] for details on this definition. Let $\operatorname{FD}(X)=F(X) \times X \times F(X)$ and, as above, consider $u \check{x} a:=(u, x, a)$, where $u, a \in F(X)$ and $x \in X$, thereby the set $\operatorname{FD}(X)$ can be denoted by $F(X) \check{X} F(X)$.

The binary maps $\dashv, \vdash$ given in [14] are now rewritten as follows:

$$
\begin{aligned}
& u \check{x} a \dashv v \check{y} b=u \check{x} a v y b \\
& u \check{x} a \vdash v \check{y} b=u x a v \check{y} b
\end{aligned}
$$

for all $u, v, a, b \in F(X)$ and $x, y \in X$. It is not hard to prove that the set $\operatorname{FD}(X)$ together with the binary operations $\dashv, \vdash$ is a g-digroup. The set of bar units of $\operatorname{FD}(X)$ is given by

$$
E(X)=\{v \check{y} b \mid v y b=e\}
$$

and its inverses are of the form

$$
\begin{aligned}
& (u \check{x} a)_{l_{(v \check{y} b)}^{-1}}^{-1}=v \check{y} b a^{-1} x^{-1} u^{-1}=v \check{y} b(u x a)^{-1}, \\
& (u \check{x} a)_{r_{(v \check{y} b)}}^{-1}=a^{-1} x^{-1} u^{-1} v \check{y} b=(u x a)^{-1} v \check{y} b,
\end{aligned}
$$

where $e$ is the unit of the free group $F(X)$ and $w^{-1}$ is the inverse of $w$ in $F(X)$.

Theorem 4. ([14]) Let $X$ be a countable set. Then $\operatorname{FD}(X)$ is a free g-digroup, with $i: X \hookrightarrow \mathrm{FD}(X)$ given by $i(x)=\check{x}$.

Proof. Let $f: X \rightarrow D$ be a set function from $X$ into a g-digroup $D$. For a fixed bar unit $\xi$ in $D$, consider the function $f_{\xi}: X \rightarrow G_{l}^{\xi}$, where $f_{\xi}(x)=\xi \dashv f(x)$. The well definition of this function comes directly from Theorem 1. Thus, there exists a group homomorphism $\varphi_{f_{\xi}}: F(X) \rightarrow G_{l}^{\xi}$, such that $\varphi_{f_{\xi}}(x)=\xi \dashv f(x)$ and $\varphi_{f_{\xi}}(e)=\xi$. It is clear that, for every reduced word $x_{i_{1}}^{\delta_{1}} \cdots x_{i_{n}}^{\delta_{n}}$ in $F(X)$

$$
\varphi_{f_{\xi}}\left(x_{i_{1}}^{\delta_{1}} \cdots x_{i_{n}}^{\delta_{n}}\right)=\xi \dashv f\left(x_{i_{1}}\right)^{\delta_{1}} \dashv \cdots \dashv f\left(x_{i_{n}}\right)^{\delta_{n}},
$$

where

$$
f\left(x_{i_{j}}\right)^{\delta_{j}}=\left\{\begin{array}{ccc}
f\left(x_{i_{j}}\right) \dashv \cdots \dashv f\left(x_{i_{j}}\right) \quad\left(\delta_{j} \text {-times }\right) & \text { if } \quad \delta_{j}>0 \\
f\left(x_{i_{j}}\right)_{l_{\xi}}^{-1} \dashv \cdots \dashv f\left(x_{i_{j}}\right)_{l_{\xi}}^{-1} \quad\left(-\delta_{j} \text {-times }\right) \quad \text { if } \quad \delta_{j}<0
\end{array} .\right.
$$


Now, we can define the function $\varphi_{\xi}: \operatorname{FD}(X) \rightarrow D$ as follows

$$
\varphi_{\xi}(u \check{x} v)=\varphi_{f_{\xi}}(u) \vdash f(x) \dashv \varphi_{f_{\xi}}(v) .
$$

Thereby, if $u=x_{i_{1}}^{\delta_{1}} \cdots x_{i_{n}}^{\delta_{n}}$ and $v=y_{t_{1}}^{\beta_{1}} \cdots y_{t_{m}}^{\beta_{m}}$ in $F(X)$, we have that

$$
\varphi_{\xi}(u \check{x} v)=f\left(x_{1_{i}}\right)^{\delta_{1}} \vdash \cdots \vdash f\left(x_{i_{n}}\right)^{\delta_{n}} \vdash f(x) \dashv f\left(y_{t_{1}}\right)^{\beta_{1}} \cdots f\left(y_{t_{m}}\right)^{\beta_{m}} .
$$

Therefore, from Proposition $1, \varphi_{\xi}$ does not depend on the choice of the bar unit $\xi$, so we denote $\varphi_{\xi}$ by $\varphi$. The proof of the fact that $\varphi$ is a gdigroup homomorphism is done in [14, Theorem 7]. The proof ends with the following equality

$$
\varphi(\check{x})=\xi \vdash f(x) \dashv \xi=f(x) .
$$

It can be easily proven that if $D$ is another free g-digroup on the set $X$, then $D$ is isomorphic to $\operatorname{FD}(X)$. Thus, up to isomorphisms, $\operatorname{FD}(X)$ is unique.

Let us consider the subset $\check{X} \subset \mathrm{FD}(X)$ of all $\check{x}:=e \check{x} e, x \in X$. From the equations (5) and (6), we have that

$$
\check{x}_{l_{x^{-1}}}^{-1}=x^{-1} \check{x} x^{-1}=\check{x}_{r_{\check{x} x}-1}^{-1} .
$$

In this way we define, for all $x \in X$ and $n \in \mathbb{Z}$,

$$
\check{x}_{\vdash}^{n}=\left\{\begin{array}{cc}
\check{x} \vdash \cdots \vdash \check{x}=x^{n-1} \check{x} & \text { if } \quad n>0 \\
\check{x}_{l_{x^{-1}}-1}^{-1} \vdash \cdots \vdash \check{x}_{l_{x^{-1}}}^{-1}=x^{n} \check{x} x^{-1} & \text { if } \quad n<0
\end{array} .\right.
$$

Similarly, we define $\check{x}_{\dashv}^{n}$. Thus, the following two equalities arise.

$$
\check{x}_{\dashv}^{n} \vdash w=\check{x}_{\vdash}^{n} \vdash w \quad \text { and } \quad w \dashv \check{x}_{\dashv}^{n}=w \dashv \check{x}_{\vdash}^{n} .
$$

Therefore, for the proof of the following proposition we use $\check{x}^{n}$ instead $\check{x}_{\vdash}^{n}$. In Proposition 2 of [22], one class of generalized digroups is constructed which, under certain conditions, gives free generalized digroups. Indeed, if in Proposition 2 of [22] suppose that $n=3$ and $G=F\left(X_{2}\right)$, where $F\left(X_{2}\right)$ is the free group on $X_{2}$, we obtain the construction $F\left(X_{2}\right) \times X_{2} \times F\left(X_{2}\right)$ of the free generalized digroup.

Proposition 2. The free g-digroup $\operatorname{FD}(X)$ is generated by the set $\check{X}$. 
Proof. Let $u \check{x} v \in \mathrm{FD}(X)$. Then, there exist $g_{1}, \cdots, g_{p}, h_{1}, \cdots, h_{k} \in X$ and $n_{i} \in \mathbb{Z}, i=1, \cdots, p$ and $m_{j} \in \mathbb{Z}, j=1, \cdots, k$, such that

$$
u \check{x} v=g_{1}^{n_{1}} \cdots g_{p}^{n_{p}} \check{x} h_{1}^{m_{1}} \cdots h_{k}^{m_{k}} .
$$

It is not hard to prove that

$$
u \check{x} v=\check{g}_{1}^{n_{1}} \vdash \ldots \vdash \check{g_{p}}{ }^{n_{p}} \vdash \check{x} \dashv{\check{h_{1}}}^{m_{1}} \dashv \ldots \dashv \check{h_{k}}{ }^{m_{k}} .
$$

As a consequence, $\operatorname{FD}(X)$ is generated by $\check{X}$.

Let $X$ be a non empty set and let $\operatorname{Sym}(X)$ denote the symmetric group on the set $X$. Let $\operatorname{Aut}(\operatorname{FD}(X))$ be the set of all bijective g-digroup homomorphisms from $\operatorname{FD}(X)$ onto itself.

Theorem 5. The group $\operatorname{Sym}(X)$ is isomorphic to a subgroup of $\operatorname{Aut}(\mathrm{FD}(X))$.

Proof. Let $f$ be an element of $\operatorname{Sym}(X)$. Then, $\check{f}: \check{X} \rightarrow \operatorname{FD}(X)$, defined by $\check{f}(\check{x})=\check{y}$, where $y=f(x)$ extends to an unique g-digroup homomorphism $\varphi_{f}: \operatorname{FD}(X) \rightarrow \operatorname{FD}(X)$, such that $\varphi_{f}(\check{x})=\check{f}(\check{x})$. In order to make the notation easier, we use $\breve{f}(x)$ instead $\check{y}$, for the case when $y=f(x)$.

Let $u \check{x} v \in \mathrm{FD}(X)$, from the proof of Proposition 2, there are $g_{1}, \cdots, g_{p}$, $h_{1}, \cdots, h_{k} \in X$ and $n_{i} \in \mathbb{Z}, i=1, \cdots, p$ and $m_{j} \in \mathbb{Z}, j=1, \cdots, k$, such that $u \check{x} v=\check{g}_{1}^{n_{1}} \vdash \ldots \vdash \check{g_{p}}{ }^{n_{p}} \vdash \check{x} \dashv \check{h}_{1}^{m_{1}} \dashv \ldots \dashv{\check{h_{k}}}^{m_{k}}$. Thus, we have that

$$
\begin{aligned}
\varphi_{f}(u \check{x} v) & =\varphi_{f}\left(\check{g}_{1}^{n_{1}} \vdash \cdots \vdash \check{g_{p}}{ }^{n_{p}} \vdash \check{x} \dashv \check{h_{1}}{ }^{m_{1}} \dashv \cdots \dashv \check{h_{k}}{ }^{m_{k}}\right) \\
& =\varphi_{f}\left(\check{g_{1}}\right)^{n_{1}} \vdash \cdots \vdash \varphi_{f}\left(\check{g_{p}}\right)^{n_{p}} \vdash \varphi_{f}(\check{x}) \dashv \varphi_{f}\left(\check{h_{1}}\right)^{m_{1}} \dashv \cdots \dashv \varphi_{f}\left(\check{h_{k}}\right)^{m_{k}} \\
& =\check{f}\left(g_{1}\right)^{n_{1}} \vdash \cdots \vdash \check{f}\left(g_{p}\right)^{n_{p}} \vdash \check{f}(x) \dashv \check{f}\left(h_{1}\right)^{m_{1}} \dashv \cdots \dashv \check{f}\left(h_{k}\right)^{m_{k}} \\
& =f\left(g_{1}\right)^{n_{1}} \cdots f\left(g_{p}\right)^{n_{p}} \check{f}(x) f\left(h_{1}\right)^{m_{1}} \cdots f\left(h_{k}\right)^{m_{k}},
\end{aligned}
$$

where

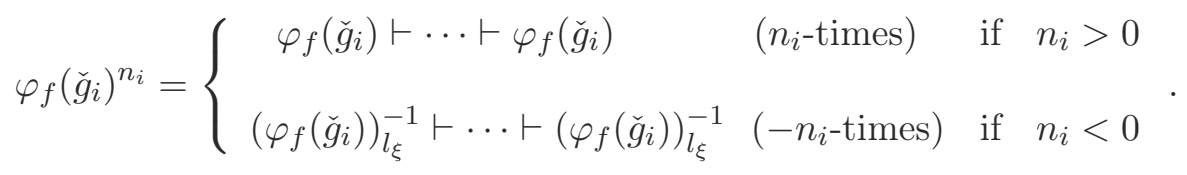

A similar equality is true for $\varphi_{f}\left(\check{h}_{j}\right)^{m_{j}}$.

Consider another function $\lambda \in \operatorname{Sym}(X)$, then

$$
\varphi_{\lambda}(u \check{x} v)=\lambda\left(g_{1}\right)^{n_{1}} \cdots \lambda\left(g_{p}\right)^{n_{p}} \lambda \check{\lambda}(x) \lambda\left(h_{1}\right)^{m_{1}} \cdots \lambda\left(h_{k}\right)^{m_{k}} .
$$


Thereby, $\varphi_{f} \circ \varphi_{\lambda}=\varphi_{f \circ \lambda}$. Moreover, if $1_{X}$ is the identity function in $\operatorname{Sym}(X)$, then $\varphi_{1_{X}}: \operatorname{FD}(X) \rightarrow \operatorname{FD}(X)$ is the identity function. Thus, for every $f \in \operatorname{Sym}(X), \varphi_{f} \in \operatorname{Aut}(\operatorname{FD}(X))$. Hence, the following function

$$
\Phi: \operatorname{Sym}(X) \rightarrow \operatorname{Aut}(\operatorname{FD}(X)), \text { where } \Phi(f)=\varphi_{f}
$$

is well defined and it is a group homomorphism. Let $f, \lambda \in \operatorname{Sym}(X)$ such that $\Phi(f)=\Phi(\lambda)$, then for every $\check{x} \in \check{X}$, we have that

$$
\Phi(f)(\check{x})=\Phi(\lambda)(\check{x}) \quad \Longleftrightarrow \quad \check{f}(x)=\check{\lambda}(x) \quad \Longleftrightarrow \quad f(x)=\lambda(x) .
$$

As a consequence, $f \equiv \lambda$.

\section{3. g-digroups action and representation theory}

In this section we explore the concept of a g-digroup action from two points of view, one as a natural extension of group actions and the other by considering a pair of compatible actions. The first one is motivated by the definition of a g-digroup representation given in [14, Definition 9] and the second one from the digroup action introduced by H. Guzmán F. Ongay in [5].

\subsection{Classical actions and Burnside's formula}

We recall the definition of a g-digroup representation.

Definition 4. Let $(D, \vdash, \dashv)$ and $\left(D^{\prime}, \vdash^{\prime}, \dashv^{\prime}\right)$ be two g-digroups and let $\operatorname{Aut}(D)$ be the set of all bijective self homomorphisms of $D$. A representation of $D^{\prime}$ on $D$ is a function $\varphi: D^{\prime} \rightarrow \operatorname{Aut}(D)$, with $\varphi\left(u^{\prime}\right)=\varphi_{u^{\prime}}$, such that, for every $u^{\prime}, v^{\prime}$ in $D^{\prime}$ and every $w \in D$,

$$
\varphi_{u^{\prime} \vdash^{\prime} v^{\prime}}(w)=\varphi_{u^{\prime}}\left(\varphi_{v^{\prime}}(w)\right)=\left(\varphi_{u^{\prime}} \circ \varphi_{v^{\prime}}\right)(w)
$$

and

$$
\varphi_{u^{\prime} \dashv^{\prime} v^{\prime}}(w)=\varphi_{u^{\prime}}\left(\varphi_{v^{\prime}}(w)\right)=\left(\varphi_{u^{\prime}} \circ \varphi_{v^{\prime}}\right)(w)
$$

It is well known in group theory that every representation induces a natural group action. We propose a natural extension of such idea to g-digroups.

Definition 5 (Classical action). Let $(D, \vdash,-)$ be a g-digroup and let $M$ be a set. A classical action is a function $\diamond: D \times M \rightarrow M$, with $\diamond(x, m)=x \diamond m$, such that, for every $x, y \in D$ and $m \in M$, 
A1) $x \diamond(y \diamond m)=(x \vdash y) \diamond m$,

A2) $(x \vdash y) \diamond m=(x \dashv y) \diamond m$ and

A3) for every bar unit $e \in D, e \diamond m=m$.

In this case, $M$ is called a classical $D$-set or for simplicity, a $D$-set.

Note that that if $\vdash=-$, then $\diamond$ is a group action. An immediate consequence of the previous definition is the following proposition

Proposition 3. If $D=G \times E$ is a g-digroup and $M$ is a $D$-set, then $M$ is a G-set. Conversely, if $M$ is a G-set, then $M$ can be seen as a D-set.

Proof. For $(g, \delta) \in D$, let $*_{\delta}: G \times M \rightarrow M$, be the function $g *_{\delta} m:=$ $*_{\delta}(g, m)=(g, \delta) \diamond m$, where $\diamond$ is a classical action. Since

$$
\begin{aligned}
g *_{\delta} m & =(g, \delta) \diamond m \\
& =((e, \xi) \vdash(g, \delta)) \diamond m \\
& =((e, \xi) \dashv(g, \delta)) \diamond m \\
& =(g, \xi) \diamond m \\
& =g *_{\xi} m,
\end{aligned}
$$

we have that $*_{\xi}=*_{\delta}$, thus we use $*$ instead of $*_{\xi}$.

Let $g, h \in G$, e the identity in $G$ and $m \in M$, then

$g *(h * m)=(g, \xi) \diamond((h, \xi) \diamond m)=((g, \xi) \dashv(h, \xi)) \diamond m=(g h, \xi) \diamond m=g h * m$

and

$$
e * m=(e, \xi) \diamond m=m .
$$

Therefore, $M$ is a $G$-set under the action $*$.

Conversely, assume that $M$ is a $G$-set under the action $*$, then $\diamond$ : $D \times M \rightarrow M$, with $(g, \xi) \diamond m=g * m$, is a classical action of $D$ over $M$. In fact, let $(g, \xi)$ and $(h, \delta)$ in $D$ and $m \in M$, then

A1) $(g, \xi) \diamond((h, \delta) \diamond m)=g *(h * m)=g h * m=(g h, g \bullet \delta) \diamond m=((g, \xi) \vdash$ $(h, \delta)) \diamond m$

A2) $((g, \xi) \vdash(h, \delta)) \diamond m=g h * m=(g h, \xi) \diamond m=((g, \xi) \dashv(h, \delta)) \diamond m$ and

A3) for every $(e, \xi)$ bar unit in $D,(e, \xi) \diamond m=e * m=m$.

Hence, $M$ is a $D$-set under the classical action $\diamond$.

It is not hard to verify that $\diamond$ does not depend on the left inverse of $(g, \xi)$ chosen. In fact, it does not change if instead of $(g, \xi)_{l_{(e, \omega)}}^{-1}$ we take $(g, \xi)_{r_{(e, \omega)}}^{-1}$, see [17, Corollary 2]. 
We know that the symmetric group $\operatorname{Sym}(D)$ is a trivial g-digroup in the sense that $\dashv=\vdash$ and $\sigma \vdash \lambda=\sigma \circ \lambda$. Consider the $\operatorname{map} \varphi: D \rightarrow \operatorname{Sym}(D)$, defined by $\varphi(x)=: \varphi_{x}$, where $\varphi_{x}(y)=x \diamond y$. This map is well defined. Indeed, let $x=(g, \xi)$ and $(h, \eta),\left(h^{\prime}, \eta^{\prime}\right) \in D$, since

$$
\begin{aligned}
\varphi_{(g, \xi)}((h, \eta))=\varphi_{(g, \xi)}\left(\left(h^{\prime}, \eta^{\prime}\right)\right) & \Leftrightarrow\left(g h g^{-1}, g \bullet \eta\right)=\left(g h^{\prime} g^{-1}, g \bullet \eta^{\prime}\right. \\
& \Leftrightarrow(h, \eta)=\left(h^{\prime}, \eta^{\prime}\right),
\end{aligned}
$$

then $\varphi_{x}$ is a one to one function. Furthermore, for every $(h, \eta) \in D$,

$$
\varphi_{(h, \xi)}\left(\left(h, g^{-1} \bullet \eta\right)\right)=(h, \eta),
$$

so $\varphi_{(g, \xi)}$ is a surjective function. Thus, $\varphi$ is well defined.

Proposition 4. The function $\varphi: D \rightarrow \operatorname{Sym}(D)$ is a g-digroup homomorphism. Moreover, $\varphi$ is injective if and only if the center $Z(G)$ is trivial and $E$ has one element.

Proof. The first part is true because of the fact that $\diamond$ is a classical action of $D$ into itself. The rest of the proof follows from the equivalence

$$
(g, \xi) \diamond(h, \eta)=(\widetilde{g}, \widetilde{\xi}) \diamond(h, \eta) \Leftrightarrow\left(g h g^{-1}, g \bullet \eta\right)=\left(\widetilde{g} h \widetilde{g}^{-1}, \widetilde{g} \bullet \eta\right),
$$

for every $g, \widetilde{g}, h \in G, \xi, \widetilde{\xi}, \eta \in E$. Consequently, $\widetilde{g}^{-1} g \in Z(G)$ and $\xi=\widetilde{\xi}$, for every $\xi, \widetilde{\xi} \in E$.

Consider the function $\diamond^{\prime}: D \times D \rightarrow D$, where $x \diamond^{\prime} y=x \vdash y$ is the left translation. Then, $\diamond^{\prime}$ is a classical action. In fact,

A1) $x \diamond^{\prime}\left(y \diamond^{\prime} z\right)=x \vdash(y \vdash z)=(x \vdash y) \diamond^{\prime} z$,

A2) $(x \dashv y) \diamond^{\prime} z=(x \dashv y) \vdash z=(x \vdash y) \diamond^{\prime} z$ and

A3) $e \diamond^{\prime} z=e \vdash z=z$.

Again, let $\varphi_{x}: D \rightarrow D$, with $\varphi_{x}(y)=x \diamond^{\prime} y$. Then, if $x=(g, \xi)$ and $y=(h, \eta)$,

$$
\varphi_{(g, \xi)}(h, \eta)=(g h, g \bullet \eta) .
$$

It is straightforward to prove that $\varphi_{x} \in \operatorname{Sym}(D)$ and so $\varphi: D \rightarrow$ $\operatorname{Sym}(D)$, where $\varphi(x)=\varphi_{x}$ is well defined. Besides, $\varphi$ is a g-digroup homomorphism, indeed,

$$
\begin{aligned}
\varphi_{x \vdash y}(z) & =(x \vdash y) \diamond^{\prime} z=(x \vdash y) \vdash z=x \vdash(y \vdash z) \\
& =x \diamond^{\prime}\left(y \diamond^{\prime} z\right)=\left(\varphi_{x} \circ \varphi_{y}\right)(z)
\end{aligned}
$$


and

$$
\begin{aligned}
\varphi_{x \dashv y}(z) & =(x \dashv y) \diamond^{\prime} z=(x \dashv y) \vdash z=x \vdash(y \vdash z) \\
& =x \diamond^{\prime}\left(y \diamond^{\prime} z\right)=\left(\varphi_{x} \circ \varphi_{y}\right)(z),
\end{aligned}
$$

for every $x, y, z \in D$. However, since $\varphi_{(g, \xi)}=\varphi_{(g, \eta)}$, for every $\xi, \eta \in E, \varphi$ is not a generalized isomorphism, unless $E$ has one element.

The previous constructions motive the following definition and theorem.

Definition 6. A regular representation of a g-digroup $D$ is a digroup homomorphism from $D$ into a permutation group.

Theorem 6 (Cayley theorem). There exists a faithful regular representation of a g-digroup $D$ if and only if $D$ is a group.

It is not to hard to see, doing a similar proof as the one of the previous theorem, that there is not a generalized isomorphism from a g-digroup $D$ onto a group $G$ unless at least $D$ is itself a group.

Theorem 7. If $M$ is a $D$-set under the action $\diamond$, then

$$
\gamma: D \rightarrow \operatorname{Aut}(M), \text { with } \gamma(x)(m)=\gamma_{x}(m)=x \diamond m, m \in M,
$$

extends to an unique representation $\Psi$ of $D$ on $D^{\prime}$, where $D^{\prime}=\mathrm{FD}(M)$ is the free g-digroup on $M$. Moreover, if $\psi: D \rightarrow \operatorname{Aut}\left(D^{\prime}\right)$ is a representation, then $D^{\prime}$ can be seen as a $D$-set in the classical sense of group theory.

Proof. Suppose that $M$ is a $D$-set under an action $\diamond: D \times M \rightarrow M$. Then, for every $x \in D, \gamma_{x}: M \rightarrow M$, where $\gamma_{x}(m)=x \diamond m$, is a bijective function. From Theorem 5, $\Phi\left(\gamma_{x}\right) \in \operatorname{Aut}(\operatorname{FD}(M))$. Thus, we can define the composite function

$$
\Psi:=\Phi \circ \gamma: D \rightarrow \operatorname{Aut}(\operatorname{FD}(M)), \text { with } \Psi(x)=\varphi_{\gamma_{x}}
$$

Let $\quad w \check{m} u \in \operatorname{FD}(M)$, with $w=m_{i_{1}}^{e_{i_{1}}} m_{i_{2}}^{e_{i_{2}}} \cdots m_{i_{t}}^{e_{i_{t}}}$ and $u=$ $m_{j_{1}}^{e_{j_{1}}} m_{j_{2}}^{e_{j_{2}}} \cdots m_{j_{s}}^{e_{j_{s}}}$, free words in $\operatorname{FD}(M)$. Then, from (11),

$$
\begin{aligned}
\Psi(x)(w \check{m} u) & =\varphi_{\gamma_{x}}(w \check{m} u) \\
& \left.=\gamma_{x}\left(m_{i_{1}}\right)^{e_{i_{1}}} \cdots \gamma_{x}\left(m_{i_{t}}\right)^{e_{i_{t}}} \gamma_{x} \check{(m}\right) \gamma_{x}\left(m_{j_{1}}\right)^{e_{j_{1}}} \cdots \gamma_{x}\left(m_{j_{s}}\right)^{e_{j_{s}}}
\end{aligned}
$$


In addition,

$$
\begin{aligned}
\gamma_{x}\left(m_{i_{j}}\right)^{e_{i_{j}}} & =\left(x \diamond m_{i_{j}}\right)^{e_{i_{j}}}=: x \diamond m_{i_{j}}^{e_{i_{j}}} \text { and } \gamma_{x}\left(m_{j_{i}}\right)^{e_{j_{i}}} \\
& =\left(x \diamond m_{j_{i}}\right)^{e_{j_{i}}}=: x \diamond m_{j_{i}}^{e_{j_{i}}} .
\end{aligned}
$$

On the other hand, for every $x \in D$ and every $w \in F(X)$, we define

$$
\gamma_{x}(w):=\gamma_{x}\left(m_{i_{1}}\right)^{e_{i_{1}}} \gamma_{x}\left(m_{i_{2}}\right)^{e_{i_{2}}} \cdots \gamma_{x}\left(m_{i_{t}}\right)^{e_{i_{t}}}
$$

Equations (15) and (16) summarize the natural extension of $\diamond$ to a classical action of $D$ over the free group $F(M)$. Thus, we have the reformulation of Equation (14) as follows

$$
\left.\Psi(x)(w \check{m} u)=\gamma_{x}(w) \gamma_{x} \check{(m}\right) \gamma_{x}(u) .
$$

From (14)-(16), we have that

$$
\begin{aligned}
\Psi(x)(w \check{m} u \vdash \widehat{w} \check{n} \widehat{u}) & =\Psi(x)((w m u \widehat{w} \check{n} \widehat{u})) \\
& =\left(\gamma_{x}(w) \gamma_{x}(m) \gamma_{x}(u) \gamma_{x}(\widehat{w}) \gamma_{x} \check{(}(n) \gamma_{x}(\widehat{u})\right) \\
& =\left(\gamma_{x}(w) \gamma_{x}(m) \gamma_{x}(u)\right) \vdash\left(\gamma_{x}(\widehat{w}) \gamma_{x} \check{(}(n) \gamma_{x}(\widehat{u})\right) \\
& =\Psi(x)(w \check{m} u) \vdash \Psi(x)(\widehat{w} \check{n} \widehat{u}) .
\end{aligned}
$$

and

$$
\begin{aligned}
\Psi(x)(w \check{m} u) \dashv \widehat{w} \check{n} \widehat{u}) & =\Psi(x)(w \check{m} u \widehat{w} n \widehat{u}) \\
& \left.=\gamma_{x}(w) \varphi_{x} \check{(m}\right) \gamma_{x}(u) \gamma_{x}(\widehat{w}) \gamma_{x}(n) \gamma_{x}(\widehat{u}) \\
& \left.=\left(\gamma_{x}(w) \varphi_{x} \check{(m}\right) \gamma_{x}(u)\right) \dashv\left(\gamma_{x}(\widehat{w}) \varphi_{x}(n) \gamma_{x}(\widehat{u})\right) \\
& =\Psi(x)(w \check{m} u) \dashv \Psi(x)(\widehat{w} \check{n} \widehat{u}) .
\end{aligned}
$$

Moreover, since the words $\gamma_{x}(w)$ and $\gamma_{x}(\widehat{w})$, for $w=m_{i_{1}}^{e_{i_{1}}} m_{i_{2}}^{e_{i_{2}}} \cdots m_{i_{t}}^{e_{i_{t}}} \in$ $F(M)$ and $\widehat{w}=m_{l_{1}}^{e_{l_{1}}} m_{l_{2}}^{e_{l_{2}}} \cdots m_{l_{r}}^{e_{l_{r}}} \in F(M)$, are also free words in $F(M)$, then the assumption $\gamma_{x}(w)=\gamma_{x}(\widehat{w})$, implies $t=s$ and, up to rearrangement of the respective letters, $\gamma_{x}\left(m_{i_{v}}\right)=\gamma_{x}\left(m_{l_{v}}\right)$, so $m_{i_{v}}=m_{l_{v}}$, for every $v=1, \cdots, t$. Thereby, $\Psi(x) \in \operatorname{Aut}(\operatorname{FD}(M))$.

Let $x, y \in D$, from (15) and (16)

$$
\begin{aligned}
\gamma_{x \vdash y}(w) & =\left((x \vdash y) \diamond m_{i_{1}}^{e_{i_{1}}}\right)\left((x \vdash y) \diamond m_{i_{2}}^{e_{i_{2}}}\right) \cdots\left((x \vdash y) \diamond m_{i_{t}}^{e_{i_{t}}}\right) \\
& =\left(( x \diamond ( y \diamond m _ { i _ { 1 } } ^ { e _ { i _ { 1 } } } ) ) \left(( x \diamond ( y \diamond m _ { i _ { 2 } } ^ { e _ { i _ { 2 } } } ) ) \cdots \left(\left(x \diamond\left(y \diamond m_{i_{t}}^{e_{i_{t}}}\right)\right)\right.\right.\right. \\
& =\gamma_{x}\left(\left(y \diamond m_{i_{1}}^{e_{i_{1}}}\right)\left(y \diamond m_{i_{2}}^{e_{i_{2}}}\right) \cdots\left(y \diamond m_{i_{t}}^{e_{i_{t}}}\right)\right) \\
& =\gamma_{x}\left(\gamma_{y}\left(m_{i_{1}}^{e_{i_{1}}} \cdots m_{i_{t}}^{e_{i_{t}}}\right)\right) \\
& =\gamma_{x}\left(\gamma_{y}(w) .\right.
\end{aligned}
$$


Therefore,

$$
\begin{aligned}
\Psi(x \vdash y)(w \check{m} u) & =\left(\gamma_{x \vdash y}(w) \gamma_{x \vdash y}(m) \gamma_{x \vdash y}(u)\right) \\
& =\left(\gamma_{x}\left(\gamma_{y}(w)\right), \gamma_{x}\left(\varphi_{y}(m)\right), \gamma_{x}\left(\gamma_{y}(u)\right)\right) \\
& =\Psi(x)\left(\gamma_{y}(w) \gamma_{y}(m) \gamma_{y}(u)\right) \\
& =\Psi(x)(\Psi(y)(w \check{m} u)),
\end{aligned}
$$

thus, $\Psi: D \rightarrow \operatorname{Aut}(\mathrm{FD}(M))$, is a representation. Let $\widehat{\Psi}$ another representation of $D$ on $\operatorname{FD}(M)$, such that $\left.\widehat{\Psi}(x)(w \check{u} v)=\left(\gamma_{x}(w) \varphi_{x} \check{(} u\right) \gamma_{x}(v)\right)$, for every $w, u, v \in M$. Then $\widehat{\Psi} \equiv \Psi$.

The reciprocal comes directly from the fact that $x \diamond m:=\Psi(x)(m)$ is a classical action of $D$ over $\operatorname{FD}(M)$.

Now, we introduce the notions of orbits and stabilizers, natural concepts associated to actions.

Definition 7. Let $M$ be a $D$-set under the action $\diamond$. The orbit of $m \in M$ is the set

$$
\mathcal{O}_{m}^{D}=\{x \diamond m \mid x \in D\} .
$$

The set of all elements in $D$ that leave $m$ fixed is called the stabilizer of $m$ and denoted by $\operatorname{Stab}_{D}(m)$.

We have the following theorem.

Theorem 8. With the above notation. Let $D=G \times E$ be a g-digroup and $M$ be a D-set. Then,

(a) the family $\mathcal{F}=\left\{\mathcal{O}_{m}^{D} \mid m \in M\right\}$ is a partition of $M$,

(b) $\operatorname{Stab}_{D}(m)$ is a g-subdigroup of $D$,

(c) $\mathcal{O}_{m}^{D}=\mathcal{O}_{m}^{G}$, where $\mathcal{O}_{m}^{G}$ is the orbit of $m$ under the action * and

(d) $\operatorname{Stab}_{D}(m)=\operatorname{Stab}_{G}(m) \times E$, where $\operatorname{Stab}_{G}(m)$ is the stabilizer subgroup of $m$ under the action $*$.

Proof. (a) Let $k \in \mathcal{O}_{m}^{D} \cap \mathcal{O}_{n}^{D}$, then there exist $x, y \in D$, such that $x \diamond m=$ $y \diamond n$. Thus, $m=\left(x_{l_{e}}^{-1} \dashv y\right) \diamond m \in \mathcal{O}_{n}^{D}$. In a similar way we prove that $n \in \mathcal{O}_{m}^{D}$. Therefore, $\mathcal{O}_{m}^{D}=\mathcal{O}_{n}^{D}$. The equality $M=\cup_{n \in M} \mathcal{O}_{m}^{D}$ comes from the definition of orbits.

(b) Let $x, y \in \operatorname{Stab}_{D}(m)$, then $(x \vdash y) \diamond m=x \diamond(y \diamond m)=m$ and $(x \dashv y) \diamond m=x \diamond(y \diamond m)=m$, so $\operatorname{Stab}_{D}(m)$ is closed under $\vdash$ and $\dashv$, moreover, $\operatorname{Stab}_{D}(m)$ contains the halo of $D$. If $x_{l_{e}}^{-1}$ and $x_{r_{e}}^{-1}$ are the left and right inverses of $x \in \operatorname{Stab}_{D}(m)$ respect to the bar unit $e$, then

$$
x_{l_{e}}^{-1} \diamond m=x_{l_{e}}^{-1} \diamond(x \diamond m)=\left(x_{l_{e}}^{-1} \dashv x\right) \diamond m=e \diamond m=m
$$


and

$$
\begin{aligned}
x_{r_{e}}^{-1} \diamond m & =x_{r_{e}}^{-1} \diamond(x \diamond m) \\
& =\left(x_{r_{e}}^{-1} \vdash x\right) \diamond m \\
& =\left(\left(x_{l_{e}}^{-1} \vdash e\right) \vdash x\right) \diamond m \\
& =\left(\left(x_{l_{e}}^{-1} \dashv e\right) \vdash x\right) \diamond m \\
& =\left(x_{l_{e}}^{-1} \vdash x\right) \diamond m \\
& =\left(x_{l_{e}}^{-1} \dashv x\right) \diamond m=m .
\end{aligned}
$$

As a consequence $\operatorname{Stab}_{D}(m)$ is a g-subdigroup of $D$.

(c)

$$
\begin{aligned}
\mathcal{O}_{m}^{D} & = & & \{(g, \xi) \diamond m \mid(g, \xi) \in F\} \\
& ={ }^{\text {Eq. }}(13) & & \{(g, \xi) \diamond m \mid g \in G\} \\
& = & & \{g * m \mid g \in G\}=\mathcal{O}_{m}^{G} .
\end{aligned}
$$

(d)

$$
\begin{aligned}
\operatorname{Stab}_{D}(m) & = & & \{(g, \xi) \in D \mid(g, \xi) \diamond m=m\} \\
& =\text { Eq. (13) } & & \{g \in G \mid(g, \xi) \diamond m=m\} \times E \\
& = & & \{g \in G \mid g * m=m\} \times E \\
& = & & \operatorname{Stab}_{G}(m) \times E .
\end{aligned}
$$

Let $D=G \times E$ be a finite g-digroup and $M$ be a $D$-set. We define the index of $\operatorname{Stab}_{G}(m)$ in $D$ as the rational number $\left[D: \operatorname{Stab}_{D}(m)\right]=$ $\frac{|D|}{\left|\operatorname{Stab}_{D}(m)\right|}$. Thus, from the previous theorem, $\left[D: \operatorname{Stab}_{D}(m)\right]=[G:$ $\left.\operatorname{Stab}_{G}(m)\right]$, therefore, we have the following equation

$$
\left[D: \operatorname{Stab}_{D}(m)\right]=\left|\mathcal{O}_{m}^{D}\right| \text {. }
$$

The proof of the following lemma is a direct consequence of Equation (13), then we omit it.

Lemma 1. Let $M_{(g, \xi)}$ be the set of all $m \in M$ fixed by $(g, \xi)$. Then $M_{(g, \xi)}=M_{(g, \eta)}$, for every $\xi, \eta \in E$ and $g \in G$. Thus, $M_{(g, \xi)}=M_{g}$, where $M_{g}$ is the set of all $m \in M$ fixed by $g$ under the action $*$.

We end this section with the following version of Burnside's formula. As we note, its proof is the same as the one for groups.

Theorem 9 (Burnside's formula for g-digroup actions). Let $D=G \times E$ be a finite g-digroup and let $M$ be a D-set. If $r$ is the number of orbits in $M$ under $D$, then

$$
r|D|=\sum_{(g, \xi) \in D}\left|M_{(g, \xi)}\right| .
$$


Proof. Let $N$ be the number of pairs $((g, \xi), m)$ such that $(g, \xi) \diamond m=m$, then

$$
N=\sum_{(g, \xi) \in D}\left|M_{(g, \xi)}\right| .
$$

On the other hand, for $m \in M$, there are $\left|\operatorname{Stab}_{D}(m)\right|$ pairs $((g, \xi), m)$ such that $(g, \xi) \diamond m=m$, then

$$
N=\sum_{m \in M}\left|\operatorname{Stab}_{D}(m)\right| .
$$

From Equation (17),

$$
\begin{aligned}
\sum_{m \in M}\left|\operatorname{Stab}_{D}(m)\right| & =\sum_{m \in M}|D| /\left|\mathcal{O}_{m}^{D}\right| \\
& =|D| \sum_{m \in M} 1 /\left|\mathcal{O}_{m}^{D}\right| .
\end{aligned}
$$

If $\mathcal{O} \subset M$ is an orbit, then $\sum_{m \in \mathcal{O}} 1 /\left|\mathcal{O}_{m}^{D}\right|=1$, thus

$$
N=|D| r .
$$

Hence, from Equations (18) and (19), we have that

$$
r|D|=\sum_{(g, \xi) \in D}\left|M_{(g, \xi)}\right| .
$$

A more direct proof of Burnside's formula can be gotten by applying, directly, Burnside's formula for groups.

\section{2. g-digroup actions}

In this section we explore, among other things, an extension of digroup actions proposed by Guzmán and Ongay in [5] to g-digroup actions.

Definition 8 ( $d$-equivariant). Let $D=G \times E$ be a g-digroup and $M$ be a $G$-set with action $*$. A function $\varphi: E \times M \rightarrow M$ is called $d$-equivariant if for every $g \in G, \xi$ and $\delta$ in $E$ and $m \in M$,

(a) $\varphi(g \cdot \xi, g * m)=g * \varphi(\xi, m) \quad$ (equivariant), and

(b) $\varphi(\xi, \varphi(\delta, m))=\varphi(\xi, m)$ (idempotent).

The following definition is equivalent to the one given in [5, Definition 3].

Definition 9 (g-digroup action). Let $D$ be a g-digroup. A set $M$ is said to be a $D$-set if there exist two functions

$$
\triangleleft, \triangleright: D \times M \rightarrow M,
$$

such that, for all $x, y \in D$ and $m \in M$, 
1) $x \triangleright(y \triangleright m)=(x \vdash y) \triangleright m$,

2) $x \triangleleft(y \triangleleft m)=(x \dashv y) \triangleleft m$,

3) there exists a bar unit $e \in D$, such that $e \triangleright m=m$,

4) $x \triangleright(y \triangleleft m)=(x \vdash y) \triangleleft m$ and

5) $x \triangleleft(y \triangleright m)=(x \dashv y) \triangleleft m$.

Any couple of functions $\triangleright$ and $\triangleleft$ that satisfies 1)-5) is called left-action or, for simplicity, action of $D$ over $M$.

Since, for every $x, y \in D$ and every $m \in M$,

$$
\begin{aligned}
(x \dashv y) \triangleright m & \stackrel{3)}{=}(x \dashv y) \triangleright(e \triangleright m) \\
& \stackrel{1)}{=}((x \dashv y) \vdash e) \triangleright m \\
= & ((x \vdash y) \vdash e) \triangleright m \\
& \stackrel{1)}{=} \quad(x \vdash y) \triangleright(e \triangleright m) \\
& \stackrel{3)}{=}(x \vdash y) \triangleright m,
\end{aligned}
$$

condition 3 ) is equivalent to

$\left.3^{\prime}\right)$ For every bar unit $\widehat{e} \in D$ and every $m \in M, \widehat{e} \triangleright m=m$. This is because, if we assume 3 ) and $\widehat{e}$ is a bar-unit in $D$, then

$$
\widehat{e} \triangleright m=(\widehat{e} \dashv e) \triangleright m \stackrel{(21)}{=}(\widehat{e} \vdash e) \triangleright m=e \triangleright m=m .
$$

The left side in conditions 1), 2), 4) and 5) corresponds to the four choices of couples $(\triangleright, \triangleright),(\triangleleft, \triangleleft),(\triangleright, \triangleleft)$ and $(\triangleleft, \triangleright)$. The right side of these conditions, at least for the cases 1) and 2) is very natural, but for the cases 4) and 5) we might think that we can consider other possibilities. Some of these possibilities imply that $\triangleleft=\triangleright$, i. e., the g-digroup action becomes a classical action.

An example of the previous analysis is summarized in the following proposition.

Proposition 5. With the above notation.

(a) If in the action definition, we change condition 4) by

$$
x \triangleright(y \triangleleft m)=(x \vdash y) \triangleright m .
$$

Then, $\triangleleft=\triangleright$.

(b) Moreover, if in the action definition, we change condition 5) by

$$
x \triangleleft(y \triangleright m)=(x \dashv y) \triangleright m .
$$

Then, $\triangleleft=\triangleright$. 
Proof. (a) Let $x, y \in D$ and $m \in M$ such that $x \triangleright(y \triangleleft m)=(x \vdash y) \triangleright m$, then

$$
\begin{aligned}
x \triangleright m & =(e \vdash x) \triangleright m \\
& =e \triangleright(x \triangleleft m) \\
& =x \triangleleft m .
\end{aligned}
$$

So, we have that $\triangleleft=\triangleright$.

(b) Let $x, y \in D$ and $m \in M$ such that $x \triangleleft(y \triangleright m)=(x \dashv y) \triangleright m$. Thus,

$$
\begin{aligned}
x \triangleright m & =(x \dashv e) \triangleright m \\
& =x \triangleleft(e \triangleright m) \\
& =x \triangleleft m .
\end{aligned}
$$

Hence, we have that $\triangleleft=\triangleright$.

The following theorem shows a connection between g-digroup actions and group actions.

Theorem 10. Let $D=G \times E$ be a g-digroup. Then, every $G$-set $M$ is a $D$-set under the actions

$$
\triangleleft: D \times M \rightarrow M \text { and } \triangleright: D \times M \rightarrow M
$$

defined as follows: for every $(g, \xi) \in D$ and $m \in M$,

$$
\triangleleft((g, \xi), m)=\triangleright((g, \xi), m)=g * m,
$$

where * is the action of $G$ defined over $M$.

Conversely, if $M$ is a D-set, with actions $\triangleleft$ and $\triangleright$, then $D$ is a $G$-set under the action

$$
*_{\xi}: G \times M \rightarrow M, \text { where } *_{\xi}(g, m)=(g, \xi) \triangleright m,
$$

where $\xi \in E$.

Proof. For the first part we have to prove that the functions defined by equation (23) satisfy the action conditions. Let $(g, \xi)$ and $(h, \delta) \in D$ and let $m \in M$. Since $\triangleleft=\triangleright$ we only have to verify 1$), 3$ ) and 5 ).

1) We have

$$
\begin{aligned}
(g, \xi) \triangleright((h, \delta) \triangleright m) & =(g, \xi) \triangleright(h * m) \\
& =g *(h * m)=(g h) * m=(g h, g(\delta)) \triangleright m \\
& =((g, \xi) \vdash(h, \delta)) \triangleright m .
\end{aligned}
$$


3) The bar units of $D$ are of the form $(e, \xi)$, where $e$ is the identity of $G$. So, we have that $(e, \xi) \triangleright m=e * m=m$.

5) We have

$$
\begin{aligned}
(g, \xi) \triangleright((h, \delta) \triangleright m) & =(g, \xi) \triangleright(h * m) \\
& =g *(h * m)=(g h) * m=(g h, \xi) \triangleright m \\
& =((g, \xi) \dashv(h, \delta)) \triangleright m .
\end{aligned}
$$

Conversely, since $\triangleright$ is a function, so is $*_{\xi}$ for any $\xi \in E$. Let $g, h \in G$ and $e$ be the identity in $G$, then

$$
\begin{aligned}
g *_{\xi}\left(h *_{\xi} m\right) & =(g, \xi) \triangleright((h, \xi) \triangleright m) \\
& =((g, \xi) \vdash(h, \xi)) \triangleright m \\
& =((g, \xi) \vdash(h, \xi)) \triangleright((e, \xi) \triangleright m) \\
& =(((g, \xi) \vdash(h, \xi)) \vdash(e, \xi)) \triangleright m \\
& =(((g, \xi) \dashv(h, \xi)) \vdash(e, \xi)) \triangleright m \\
& =((g, \xi) \dashv(h, \xi)) \triangleright((e, \xi) \triangleright m) \\
& =(g h, \xi) \triangleright m \\
& =(g h) *_{\xi} m .
\end{aligned}
$$

Besides, $e *_{\xi} m=(e, \xi) \triangleright m=m$. Thus, $*_{\xi}$ is an action of $G$ over $M$.

We have the following characterization which is the same to the one given in [5], up to the definition of $d$-equivariance.

Theorem 11 (A characterization of a g-digroup action). Let $D=G \times E$ be a g-digroup and $M$ be a $G$-set under the action $*$. Then, $M$ can be endowed with a D-set structure in which $*=*_{\xi}$ is the action defined by Equation (24) if and only if there exists a d-equivariant function $\varepsilon: E \times M \rightarrow M$.

Proof. Suppose that $M$ is a $D$-set under the actions $(\triangleleft, \triangleright)$. We define the function

$$
\varepsilon: E \times M \rightarrow M
$$

where $\varepsilon(\xi, m)=(e, \xi) \triangleleft m$. Indeed,

$$
\begin{aligned}
\varepsilon(h \cdot \xi, h * m)= & (e, h \cdot \xi) \triangleleft h * m \\
= & (e, h \cdot \xi) \triangleleft((h, \xi) \triangleright m)) \\
& \stackrel{5)}{=}((e, h \cdot \xi) \dashv(h, \xi)) \triangleleft m \\
= & (h, h \cdot \xi) \triangleleft m \\
= & ((h, \xi) \vdash(e, \xi)) \triangleleft m \\
& \stackrel{4)}{=}(h, \xi) \triangleright((e, \xi) \triangleleft m) \\
= & (h, \xi) \triangleright \varepsilon(\xi, m) \\
= & h * \varepsilon(\xi, m) .
\end{aligned}
$$


Besides, $\varepsilon(\xi, \varepsilon(\eta, m))=(e, \xi) \triangleleft((e, \eta) \triangleleft m)=(e, \xi) \triangleleft m=\varepsilon(\xi, m)$. Then, we have proven that $\varepsilon$ is a $d$ - equivariant function.

Conversely, suppose that $\varepsilon: E \times M \rightarrow M$ is $d$-equivariant. We define $(g, \xi) \triangleright m=g * m$ and $(g, \xi) \triangleleft m=\varepsilon(\xi, g * m)$. Thus, we have to verify if they satisfy the g-digroup action conditions.

1) For every $(g, \xi),(h, \delta) \in D$ and $m \in M$,

$$
\begin{aligned}
(g, \xi) \triangleright((h, \delta) \triangleright m) & =g *(h * m) \\
& =(g h) * m \\
& =(g h, g \cdot \delta) \triangleright m \\
& =((g, \xi) \vdash(h, \delta)) \triangleright m .
\end{aligned}
$$

2) Let $(g, \xi),(h, \delta) \in D$ and $m \in M$, then

$$
\begin{aligned}
(g, \xi) \triangleleft((h, \delta) \triangleleft m) & =(g, \xi) \triangleleft(\varepsilon(\delta, h * m)) \\
& =\varepsilon(\xi, g * \varepsilon(\delta, h * m)) \\
& =\varepsilon(\xi, \varepsilon(g \cdot \delta, g *(h * m))) \\
& =\varepsilon(\xi, \varepsilon(g \cdot \delta,(g h) * m))) \\
& =\varepsilon(\xi,(g h) * m) \\
& =(g h, \xi) \triangleleft m \\
& =((g, \xi) \dashv(h, \delta)) \triangleleft m .
\end{aligned}
$$

3) The bar units of $D$ are of the form $(e, \xi)$, where $e$ is the identity of $G$. So, we have that, $(e, \xi) \triangleright m=e * m=m$.

4) Let $(g, \xi),(h, \delta) \in D$ and $m \in M$, then

$$
\begin{aligned}
(g, \xi) \triangleright((h, \delta) \triangleleft m) & =(g, \xi) \triangleright(\varepsilon(\delta, h * m)) \\
& =g * \varepsilon(\delta, h * m) \\
& =\varepsilon(g \cdot \delta, g *(h * m)) \\
& =(g h, g \cdot \delta) \triangleleft m \\
& =((g, \xi) \vdash(h, \delta)) \triangleleft m .
\end{aligned}
$$

5) For every $(g, \xi),(h, \delta) \in D$ and $m \in M$, we have that

$$
\begin{aligned}
(g, \xi) \triangleleft((h, \delta) \triangleright m) & =(g, \xi) \triangleleft h * m \\
& =\varepsilon(\xi, g *(h * m)) \\
& =\varepsilon(\xi,(g h) * m)) \\
& =(g h, \xi) \triangleleft m \\
& =((g, \xi) \dashv(h, \delta)) \triangleleft m .
\end{aligned}
$$

It is well known in group theory that there is a strong connection between the symmetric group and the $G$-sets. Here we have something 
similar given in Proposition 6. Before the statement of such corollary, we recall the definition of the symmetric $g$-digroup, for more details see [16].

Let $A$ be non-empty set and let $G \leqslant \operatorname{Sym}(A)$. If $X$ is a $G$-set, with action $(g, \xi) \mapsto g \bullet \xi$, we define the map

$$
\begin{array}{ccc}
T: \operatorname{Sym}(A) \times X & \rightarrow & \operatorname{End}(A \times X) \\
(\alpha, \xi) & \mapsto & T_{(\alpha, \xi)},
\end{array}
$$

whit $T_{(\alpha, \xi)}(b, \eta)=(\alpha(b), \xi), \forall(b, \eta) \in A \times X$.

Over the set $\mathcal{T}_{G}:=\left\{T_{(g, \xi)} \mid g \in G\right.$ and $\left.\xi \in X\right\}$ we define the binary maps $\dashv$ and $\vdash$, by

$$
T_{(g, \xi)} \dashv T_{(h, \eta)}:=T_{(g h, \xi)} \text { and } T_{(g, \xi)} \vdash T_{(h, \eta)}:=T_{(g h, g \bullet \eta)} .
$$

Theorem 12 (The symmetric generalized digroup). The set $\left(\mathcal{T}_{G}, \vdash, \dashv\right)$ is a (symmetric) g-digroup with halo $\mathcal{T}_{\mathbb{I}}=\left\{T_{(I d, \xi)} \mid \xi \in X\right\}$. For any bar unit $T_{(I d, e)} \in \mathcal{T}_{\mathbb{I}}$, the left and right inverses of $T_{(g, \xi)} \in \mathcal{T}_{G}$ are

$$
T_{(g, \xi)_{l}}^{-1}=T_{\left(g^{-1}, e\right)} \quad \text { and } \quad T_{(g, \xi)_{r_{e}}}^{-1}=T_{\left(g^{-1}, g^{-1} \bullet e\right)} .
$$

As it was mentioned, the following proposition asserts the connection between $g$-action and the symmetric $g$-digroups.

Proposition 6. Let $A, X$ and a $\mathcal{T}_{G}$ as previously given, then $A \times X$ is a $\mathcal{T}_{G}$-set.

Proof. It is not hard to prove that $\mathcal{T}_{G}$ is isomorphic to the g-digroup $G \times E$, where $(g, \xi) \vdash(h, \eta)=\left(g h, \phi_{g}(\eta)\right)$ and $(g, \xi) \dashv(h, \eta)=(g h, \xi)$. Consequently, we have to prove that $A \times X$ is a $G \times E$-set, but it is an immediate consequence of the fact that $A \times E$ is naturally a $G$-set and

$$
\varepsilon: E \times(A \times E) \rightarrow A \times E,
$$

where $\varepsilon(\xi,(a, \eta))=(a, \xi)$ is a $d$-equivariant function.

(a)

$$
\begin{aligned}
\varepsilon\left(\phi_{g}(\xi), g *(a, \eta)\right) & =\varepsilon\left(\phi_{g}(\xi),\left(g(a), \phi_{g}(\eta)\right)\right) \\
& =\left(g(a), \phi_{g}(\xi)\right) \\
& =g *(a, \xi)=g * \varepsilon(\xi,(a, \eta)) .
\end{aligned}
$$

(b)

$$
\varepsilon(\xi, \varepsilon(\eta,(a, \delta)))=\varepsilon(\xi,(a, \eta))=(a, \xi)=\varepsilon(\xi,(a, \delta)) .
$$

In the following definition we introduce the concepts of orbits and isotropic g-subdigroup like in [5, Definition 4]. 
Definition 10. Let $D$ be a g-digroup and let $M$ be a $D$-set. We define the $\triangleright$-orbit and the $\triangleleft$-orbit of an element $m \in M$ as the sets $\mathcal{O}_{m}^{\triangleright}=$ $\{x \triangleright m \mid x \in D\}$ and $\mathcal{O}_{m}^{\triangleleft}=\{x \triangleleft m \mid x \in D\}$, respectively. We also define $D_{m}^{l}=\{x \in D \mid x \triangleright m=m\}$ and $D_{m}^{r}=\{x \in M \mid x \triangleleft m=m\}$.

The sets $\mathcal{O}_{m}^{\triangleright}$ and $\mathcal{O}_{m}^{\triangleleft}$ are not orbits in the sense of group actions. Thereby, we could consider the orbit of $m$ as the set $\mathcal{O}_{m}^{D}=\mathcal{O}_{m}^{\triangleright} \cup \mathcal{O}_{m}^{\triangleleft}$, but it is unknown if they partition $M$.

The proof of the following proposition is equal to the one given for [5, Proposition 7], thus we omit it.

Proposition 7. The set $D_{n}^{l}$ is a g-subdigroup of $D$, called the left isotropic g-subdigroup of $D$. Moreover, $D_{m}=D_{m}^{l} \cap D_{m}^{r}$ is also a g-subdigroup, called the bilateral isotropic g-subdigroup of $D$.

\section{Acknowledgements}

The authors thank the referee for the valuable comments. The third author acknowledges to Vicerrectoría de Investigaciones, Universidad de Antioquia.

\section{References}

[1] A. Blokh. A generalization of the concept of a Lie algebra. Dokl. Akad. Nauk, volume 165: 3, pages 471-473, Russian Academy of Sciences, 1965. English translation: Soviet Math. Dokl. 6 (1965), 1450 - 1452.

[2] M. Bordemann and F. Wagemann. Global integration of Leibniz algebras. Journal of Lie Theory, 27(2):555-567, 2017.

[3] S. Covez. The local integration of Leibniz algebras. Annales de l'institut Fourier, 63(1), 2013.

[4] R. Felipe. Digroups and their linear presentations. East-West J. Math., 8(1):27-48, 2006.

[5] H. Guzmán and F. Ongay. On the concept of digroup action. Semigroup Forum, 100:461-481, 2020.

[6] D. L. Johnson. Presentations of groups. Cambridge University Press, 1997.

[7] M. K. Kinyon. Leibniz algebras, Lie racks, and digroups. J. Lie Theory, 17(1):99114, 2007.

[8] K. Liu. A class of group-like objects.

[9] J.-L. Loday. Une version non commutative des algèbres de Lie: les algèbres de Leibniz. Enseign. Math. (2), 39(3-4):269-293, 1993.

[10] J.-L. Loday. Dialgebras. 1763:7-66, 2001.

[11] J. Monterde and F. Ongay. On integral manifolds for leibniz algebras. Algebra, $2014,2014$. 
[12] J. Mostovoy. A comment on the integration of Leibniz algebras. Comm. Algebra, 41(1):185-194, 2013.

[13] F. Ongay. On the notion of digroup. Comunicaciones del CIMAT, (I-10-04), 2010.

[14] J. G. Rodríguez-Nieto, O. P. Salazar-Díaz, and R. Velásquez. Augmented, free and tensor generalized digroups. Open Mathematics, 17(1):71-88, 2019.

[15] J. G. Rodríguez-Nieto, O. P. Salazar-Díaz, and R. Velásquez. Sylow-type theorems for generalized digroups. submitted, 2019.

[16] J. G. Rodríguez-Nieto, O. P. Salazar-Díaz, and R. Velásquez. Abelian and symmetric generalized digroups. Semigroup Forum, 32021.

[17] O.P. Salazar-Díaz, R. Velásquez, and L. A. Wills-Toro. Generalized digroups. Communications in Algebra, 44:2760-2785, 2016.

[18] J.D.H. Smith. Directional algebras. Houston Journal of Mathematics, 42(1):1-22, 2016.

[19] A. V. Zhuchok. Semilattices of subdimonoids. Asian-European Journal of Mathematics, 4(2):359-371, 2011.

[20] A. V. Zhuchok. Free n-nilpotent dimonoids. Algebra Discrete Math., 16(2):299-310, 2013.

[21] A. V. Zhuchok. Free products of dimonoids. Quasigroups and Related Systems, 21(2):273-278, 2013.

[22] A. V. Zhuchok and Y. V. Zhuchok. On two classes of digroups. Sao Paulo J. Math. Sci., 11:240-252, 2017.

CONTACT INFORMATION

José Gregorio

Rodríguez-Nieto,

Olga Patricia

Salazar-Díaz

Raúl Velásquez
Escuela de Matemáticas

Universidad Nacional de Colombia

Medellín, Colombia

E-Mail(s): jgrodrig@unal.edu.co, opsalazard@unal . edu.co

Instituto de Matemáticas

Universidad de Antioquia

Medellín, Colombia

E-Mail(s): raul.velasquez@udea.edu.co

Received by the editors: 17.12 .2020

and in final form 14.08.2021. 